\title{
Impact of Living Kidney Donation on Long-Term Renal and Patient Survival: An Evolving Paradigm
}

\author{
Geir Mjøen • Hallvard Holdaas
}

Published online: 28 December 2014

(C) Springer International Publishing AG 2014

\begin{abstract}
During the last decades, there have been several studies reporting the outcome after living kidney donation. These studies have not shown any increase in mortality or end-stage renal disease. This is reassuring for potential donors. However, in these studies, kidney donors have been compared with unselected general population controls. In recent studies, kidney donors have been compared with selected healthy controls designed to simulate the donor selection process and improve quantification of the attributable impacts of donation. Also, recent studies describe donor cohorts with a longer follow-up. One of these studies found increased mortality risk, and several studies have found increased risk of end-stage renal disease occurring several years after donation. These findings have consequences for how we evaluate and select living donors. Future studies on mortality and end-stage renal disease in donors should focus on large donor cohorts with a long follow-up and appropriate healthy control groups. Cross sectional studies performed months or years after donation should focus on comparisons with controls who were healthy at the time of the donor's evaluation. Although recent studies have indicated attributable risks compared with healthy controls, absolute event rates of end-stage renal disease and/or mortality appear fairly low. Although these studies have modified our assessment of donor risk, we still promote living kidney donation.
\end{abstract}

Keywords Living kidney donors $\cdot$ Kidney donors

This article is part of the Topical Collection on Live Kidney Donation

G. Mjøen ( $\bowtie)$

Department of Nephrology, Oslo University Hospital, 0027 Oslo,

Norway

e-mail: geir.mjoen@ous-hf.no

H. Holdaas

Department of Transplant Medicine, Oslo University Hospital,

0027 Oslo, Norway

\section{Introduction}

Kidney transplantation is the best available treatment for endstage renal disease. Compared to dialysis, kidney transplantation offers better quality of life, improved long-term survival, decreased morbidity, and is cost effective for society.

In 2013, 33.6\% of kidney transplants in the US and $27 \%$ of those in Europe were from living donors [1, 2]. Living donation reduces waiting lists and provides shorter waiting time for those who receive a kidney transplant from a deceased donor.

Since the first living kidney donation in 1954 [3], safety of the donor has been of high priority. A key issue is that in general, structured lifelong donor follow-up has not been considered mandatory. Retrospective single-center studies of varying sizes have evaluated long-term survival and risk of endstage renal disease [4-13] for donors compared to the general population. While event rates in the general population provide one benchmark for framing donor outcomes, such comparisons do not address attributable risk, because members of the general population may have coexisting medical conditions such as cardiovascular disease, malignancy, diabetes, hypertension, and chronic kidney disease that would have made them ineligible for kidney donation. Lin et al. [14] wisely suggested that living kidney donors should be compared with healthy control subjects who do not have any chronic disease that would exclude living kidney donation. Thus, conducting studies with control groups from an unselected general population sample makes it harder to detect possible attributable impacts of donor nephrectomy on subsequent health.

In this review, we present the most recent literature on longterm risk of end-stage renal disease (ESRD) and mortality in kidney donors, and how this adds to previous knowledge. Based on this, we discuss how long-term risks should be interpreted, and important consequences for clinical practice and future research. 


\section{Results}

In a landmark study from 2011, Segev et al. [15] found no higher long-term mortality for live donors than for agematched and comorbidity-matched National Health and $\mathrm{Nu}-$ trition Examination Survey 1988-1994 (NHANES III) participants. This was the first study attempting to compare living donors with a control group selected to satisfy living donor health criteria at baseline. The study included 80,347 donors donating in the period 1994-2009, with median follow-up of 6.3 years. Median follow-up was shorter (2.3 years) for donors with available data on blood pressure and smoking. Median age at donation was not stated, but $49 \%$ of donors were younger than 40 years of age. During the first year of follow-up, there were 52 deaths. A control group was drawn from 9,364 participants from NHANES III, using matching with replacement. Potential limitations of this study were possible confounding from different periods of inclusion [16], and lack of power due to a combination of short follow-up time and relatively young donors, resulting in few events $[17,18]$

A study by Garg et al. found a lower risk of mortality and cardiovascular events in previous donors compared with a healthy control group derived from provincial healthcare databases [19].

A total of 2,028 donors were followed for a median of 6.5 years (maximum 17.7 years). Median age was 43 years at the time of donation. During follow-up, there were 26 major cardiovascular events and 16 deaths. Due to relatively few events, the primary outcome was a composite of time to death or first major cardiovascular event. Matched non-donors were selected from the healthiest segment of the general population. Controls were matched to donors based on demographic data. Based on information from large health administrative databases, those with any known diseases that would preclude donation were excluded. The risk of the primary outcome of death and major cardiovascular events was lower in donors than in non-donors ( 2.8 vs. 4.1 events per 1,000 person-years; hazard ratio (HR) $0.66,95 \%$ confidence interval (CI) 0.48 to $0.90)$. Repeating analyses after sorting by gender, age, time of donation, or relative with kidney disease, did not change the results.

Our Norwegian study with long-term follow-up found increased risks for all-cause mortality, cardiovascular mortality and end-stage renal disease [20]. The study included 1,901 kidney donors with a median age of 46 years, and a median follow-up time of 15.1 years. A control group was obtained from the HUNT1 study, a cross-sectional study performed in the 1980s. Donors and controls both had available data on blood pressure, smoking and body mass index in addition to demographics, improving the selection of healthy controls. Among living donors, there were 224 deaths and nine cases of ESRD during follow-up. In relative terms, the risk of all- cause mortality was increased at $1.30(95 \%$ CI $1.11-1.52)$ for donors compared with controls, with a corresponding increase in cardiovascular death. The relative risk of ESRD was greatly increased at 11.38 (4.37-29.6). Since the donors in this study were predominantly Caucasians, and closely related to the recipient $(85 \%$ biologically related, including $80 \%$ firstdegree relatives), the results of this study may extrapolate less well to non-whites and unrelated donors.

A recent study by Reese et al. evaluated mortality and ESRD in donors over 55 years of age $[21 \bullet, 22 \cdot]$. Among 5, 152 donors, 3,368 were matched 1:1 against controls from the Health and Retirement Study. Mean age was 59 years, and median follow-up was 7.8 years. There was no significant increase in mortality $(p=0.21)[21 \bullet$. In a preliminary abstract, the authors evaluated the risk of end-stage renal disease. Based on Medicare claims, the risk of ESRD was higher in previous donors (HR 7.4, 2.9-18.8) [22•]. However, this result was not reported in the final study. Although median followup was relatively short, this is of lesser importance since donors were older. Enough events would be expected to occur during observation to allow for sufficient power in statistical analyses. However, there is uncertainty whether the control group was healthy enough, since comorbidities were only ascertained through interviews. An important limitation that the authors themselves have pointed out was that controls had not undergone any physical evaluation by a doctor, so that, for example, blood pressure at time of inclusion was not known. Accordingly, controls may have had higher blood pressure than donors. This may have contributed to the relative risk in controls, making it harder to discover a possible impact from nephrectomy on mortality.

A paper by Muzaale et al. published in 2014 included 96, 217 kidney donors who donated a kidney in the time period 1994-2011. Median follow-up was 7.6 years. Donors were compared with a matched control group consisting of participants from the NHANES III survey, included in the period 1988-1994. Controls were matched for age, gender, smoking, blood pressure and body mass index. During follow-up, 99 donors $(0.10 \%)$ developed end-stage renal disease, compared to 36 ESRD events $(0.04 \%)$ in healthy matched non-donors. Based on the reported event rates, we estimate there was an approximately eight-fold increase in relative risk of end-stage renal disease in those who had donated a kidney [23••].

In addition to finding a significantly increased risk of endstage renal disease in kidney donors, the authors found a similar incidence of end-stage renal disease in both related and unrelated donors, when comparing these two groups. This is an important finding. It is well known that there is a familial clustering of ESRD [24, 25]. Skrunes et al. found increased mortality in first-degree relatives of patients with ESRD [26]. Differentiating potential effects of donation from hereditary factors is a problem in donor studies, since most donors are closely related with the recipient, and most studies do not 
include enough unrelated donors to perform separate analyses in this group.

In Muzaale et al.'s study, the different incidence of end-stage renal disease between groups was expressed only in terms of absolute risks. An editorial in the Journal of the American Medical Association (JAMA) highlighted that low absolute risk should be reassuring to prospective donors [27]. Since end-stage renal failure is extremely rare among most healthy populations, any increase in absolute risk will inevitably be quite small. However, in populations with a higher lifetime risk of ESRD at baseline, this will not be the case [28]. A limitation of the study by Muzaale et al. is the relatively short follow-up time and lack of diagnoses regarding the cause of ESRD in the donors. Also, some baseline variables, such as education and smoking, were unevenly distributed between groups. Adjusting for these variables may have influenced the estimates.

\section{Hypertension, CKD and Proteinuria}

Since the early 1970 s, studies have been performed with the aim of uncovering possible detrimental effects of living donation. Although all-cause mortality and risk of end-stage renal disease are the most important outcomes in donor studies, the surrogate outcomes of hypertension and proteinuria are also of major importance, since these are modifiable risk factors. Since donors have reduced renal mass after nephrectomy, and reduced renal function is known to be associated with hypertension and proteinuria in the general population, one would suspect these conditions to be more prevalent among donors. Both hypertension and proteinuria are known cardiovascular risk factors in the general population, and are important indications for pharmacological treatment with the aim of preventing cardiovascular disease and premature death. Another important question is whether there is further loss in renal function beyond that anticipated from normal aging, after the initial loss of renal function due to nephrectomy.

\section{Blood Pressure}

A meta-analysis by Boudville et al. published in 2006 concluded that kidney donation was associated with a $5 \mathrm{mmHg}$ increase in blood pressure above that associated with normal aging, 10 years after surgery [29]. This was in accordance with a previous meta-analysis [30]. Among 48 included studies, Boudville et al. identified ten studies with a control group. These studies formed the basis for the conclusion of the metaanalysis. All studies were cross-sectional and performed some years after donation. Controls were healthy volunteers or individuals undergoing donor evaluation, and were selected on basis of demographic data. Except for one study [31], all controls were assembled at the time of study, and were not included at the time of donor nephrectomy. The result of this meta-analysis was, as stated by the authors, "inherently limited by the quality of the primary studies." Donors with higher blood pressure were more likely to receive follow-up, which may have inflated the apparent risk in donors. On the other hand, controls were assembled from the general population, and obviously did not undergo the same rigorous testing that donors face before surgery. A group from the U.S. and Canada recently published 6-month data on 201 donors and matched controls followed since nephrectomy [32]. Though there were no differences in blood pressure, a longer follow-up time may be needed. However, this study is still ongoing and of particular interest, since the control group is followed prospectively from donation, and except for renal vascular imaging, was thoroughly examined before inclusion.

\section{Renal Function and Albuminuria}

In a parallel effort to the paper by Boudville et al., Garg et al. published a meta-analysis on the risk of proteinuria and reduced kidney function [33]. Proteinuria was higher in donors than controls ( $147 \mathrm{mg} /$ day vs. $83 \mathrm{mg} /$ day), and became more pronounced with time. After an initial decrease in renal function from nephrectomy, there was no further loss other than that associated with normal aging. Out of 62 included studies, 11 studies included a control group for comparison. Analysis of proteinuria was based on pooling three studies, with 129 donors altogether. Five studies reported having found no association between renal function at the time of assessment and time since nephrectomy, although meta-regression was not performed. The authors also examined if renal function was indeed lower after nephrectomy than in two kidney controls, which it unsurprisingly was. As in the meta analysis by Boudville et al., most of the primary studies included control groups assembled at the time of study, as opposed to the time when donor nephrectomy was performed.

Although we do not exactly know the consequences of elevated blood pressure, albuminuria and reduced renal function in previous kidney donors, it is likely that these consequences are comparable to those seen in the general population. Inspired by what is known from the two abovementioned meta-analyses, Kiberd [34] tried to simulate the impact of hypertension, reduced glomerular filtration rate and albuminuria on life expectancy and end-stage renal disease in kidney donors. The study was performed with a computer simulation based on a US population chronic kidney disease model. In a 40-year-old white male, kidney donation was projected to reduce life expectancy by 0.83 years. Albuminuria was highly associated with life expectancy, while mild increase in blood pressure seemed to have a more modest impact on risk of premature death. Furthermore, kidney donation was associated with a $0.89 \%$ increase in the cumulative 
risk of end-stage renal disease. Decrease in renal function was most associated with this outcome, while again, the association with blood pressure was modest. When comparing absolute risk increases between different demographic groups, this study found that older donors were predicted to lose less life years and face lower cumulative risks of end-stage renal disease than younger donors. This was true even if the older donors had pre-existing hypertension.

The study by Kiberd is based on computer simulation assuming two premises. Firstly, the study assumes that nephrectomy increases blood pressure and albuminuria, and leads to reduced renal function. Secondly, it assumes that these changes have the same impact in kidney donors as in the general population. However, these assumptions may be associated with some degree of uncertainty.

Studies on blood pressure, albuminuria and renal function after donation have several limitations. Furthermore, studies from the general population estimating increases in mortality in association with these risk factors include individuals who are less healthy than kidney donors. They may have other coexisting diseases associated with hypertension or kidney disease. Although there is little discrepancy between this study and recent observational studies [20, 23••], one should interpret the results of such a simulation in light of the limitations inherently associated with such studies.

\section{Interpreting Risks}

Since recent studies have found increased risks in relation to kidney donation, the interpretation of these risks are even more relevant than previous conclusions from donors vs. general population comparisons showing that living donors live longer.

In light of what is known from the general population, and from studies in kidney donors, different donors will have different long-term risks of ESRD and premature death based on baseline age, gender, race or the occurrence of isolated medical abnormalities. Such an abnormality could be mild hypertension, low-normal renal function, microalbuminuria, nephrourolithiasis, impaired glucose tolerance or obesity. There are several aspects to consider when evaluating longterm risk, as described by Steiner [35-41]. Firstly, the potential donor's lifetime risk at baseline must be considered [37]. Secondly, the incremental risk incurred by donor nephrectomy should be taken into consideration [39]. Diseases that may be contracted later in life, such as diabetes, hypertension or primary kidney disease, may worsen the remaining renal function and lead to symptomatic renal disease at an earlier time than in a similar individual with two kidneys [35]. Reduced renal function in the donor may be a risk factor for other diseases, most importantly cardiovascular disease [20]. This association is known from studies in chronic kidney disease populations [42].
Many diseases relevant to donors are developed after the fifth or sixth decades. Accordingly, a normal donor evaluation is more reassuring in an older donor than in a younger donor [37]. Since older people in general tend to have more diseases than younger individuals, an older donor with a normal evaluation is relatively healthier than a similar younger donor. Many conditions such as diabetes or hypertension have yet to occur in a younger donor. Finally, a younger donor will spend more remaining years with only one kidney. When evaluating long-term risks based on these facts, one may infer that remaining cumulative lifetime risk is higher in a healthy 25 -year-old male than in a 60-year-old otherwise healthy male with mild hypertension [37, 38]. Likewise, basic demographic factors of age and gender may have more impact on baseline risk than the occurrence of isolated medical abnormalities, e.g., mild hypertension [38]. Presenting risks to potential donors could be performed by presenting rough estimates of absolute baseline risks and absolute risk increases [40]. Many authors emphasize the importance of absolute instead of relative risks [20, 23••]. This is especially important for the outcome of end-stage renal disease, since this is a rare outcome. In an individual with a low baseline lifetime risk, such as a middle-age healthy white female donor, even a high relative risk would be converted into a small absolute risk increase, due to the rarity of the outcome. Some have stated that the low absolute risk increase should be viewed as reassuring for prospective donors [27]. However, in those donors where remaining lifetime risk of ESRD is less than negligible; for example, a young overweight black male with a projected lifetime risk of ESRD at baseline as high as $2-3 \%$, a relative risk of approximately ten would perhaps not seem reassuring. It is also important to educate donors regarding how nephrectomy could impact their lives if they were to develop chronic kidney disease later in life. Most likely, the consequence of nephrectomy would be that the need for dialysis would arise a few years earlier than with two kidneys [35], rather than a question of whether they would develop kidney disease or not. Also, in healthy populations with a low mortality rate, a $30 \%$ increase in all-cause mortality [20] in a healthy 50-year-old kidney donor would translate into a 3year shorter life-span [43], or even less [34]. Whether hereditary factors affect the magnitude of risk is uncertain. Most studies have only 20-30\% unrelated donors included in the study population, precluding subgroup analyses. However, in a study with adequate power for subgroup analyses, risks of ESRD were similar for unrelated donors [23••].

\section{Consequences for Donor Evaluation and Donor Selection}

Acknowledgement by the transplant community that living kidney donation is associated with long-term risks is likely to have consequences for the evaluation and selection of future 
donors. Evaluation of donors is a thorough procedure. The medical evaluation is extensive [44]. However, there are some differences between centers regarding the assessment of renal function. Most centers accept donors as long as they have absolute measured glomerular filtration rate above a set limit of $70 \mathrm{or} 80 \mathrm{ml} / \mathrm{min}$ [44]. Others have suggested that the focus should be to predict the donor's post-donation estimated glomerular filtration rate normalized for body surface area [45]. Also, there are differences in how donor renal function is measured. Since post nephrectomy renal function seems to be related to increased morbidity and mortality in donors, an improvement in the precision of measuring pre-donation renal function seems warranted. It is our view that isotopemeasured glomerular filtration rate should be mandatory. It is especially important to discover young donors with lownormal renal function, as these donors would have a higher long-term risk for symptomatic renal disease, which would be further increased by nephrectomy. Furthermore, transplant centers should try to present the donor with rough risk estimates based on recent literature, and should not inform donors that their long-term survival is better than that of the background population, as this is an outdated comparison and irrelevant for the donor's decision making.

Donor selection is based on immunological fit, relation to the recipient and evaluation of donor risk. There are many considerations to be taken, and although there may be guidelines, each scenario is unique, relying on the ethical and clinical judgment of the transplant team of whether to proceed with the surgery. However, in light of recent studies, nephrologists should, to a higher degree than previously, inform about absolute donor risk during the donor's remaining lifespan. Older donors with isolated medical abnormalities should not be excluded from donation without thorough consideration of these absolute risks. One will find that in many cases, the donor with the lowest absolute future risk is the oldest. With a mean live transplant kidney survival of around 20 years [46], a younger potential donor may be given the opportunity to donate at a later date. In a time when many populations in the western world have a high lifetime risk of diabetes and cardiovascular disease, one or two decades of observations before again being considered as a donor will only be reassuring.

\section{Consequences for Future Research}

Recent findings of increased blood pressure, increased albuminuria, increased incidence of end-stage renal disease, and increased mortality need corroboration in future studies. However, to add to existing knowledge, such studies should not be underpowered. Ideally, they should include several thousand donors who are followed for more than a decade. Adequately selected control groups are indeed essential. Controls should ideally be recruited from the same population as donors at the time of donation, and should have undergone thorough physical and biochemical evaluation. Ideally, they should be healthy enough themselves to donate a kidney. If the control group is not healthy enough at baseline, this may decrease the possibility of detecting short-term and long-term risks associated with donation. A well-selected control group is crucial when performing studies of ESRD and mortality, but also when performing cross-sectional studies on hypertension, renal function and other biochemical parameters. In this type of study, finding an appropriate control group is especially difficult. The control group should have been healthy at the time of the donor's evaluation and not necessarily at the time of study. For example, if we were to conduct a cross-sectional study evaluating blood pressure in a group of kidney donors who donated in 2003, we would like to include controls who were of similar health in 2003. Including controls who are healthy today or controls from the unselected general population would both introduce bias.

\section{Conclusions}

Recent literature has advanced the field of living kidney donation. There is evidence indicating that donors face increased risks of ESRD compared with healthy nondonors. Data regarding donation-related risks of cardiovascular and all-cause mortality have been identified in one recent study. Interpretation of the impact of these studies is important, and may affect future donor evaluation, selection and follow-up after donation. There may be more rationale for selecting older donors with isolated medical abnormalities than previously thought. All potential donors will need information regarding possible long-term risks. Our summary of recent studies has shown the importance of control groups selected for baseline good health in efforts to quantify the risks attributable to donation. There is a need for future studies to corroborate the findings of donation-related risks. These studies should be adequately powered and should include highly selected control groups. Although the climate has changed regarding risks after donation, we still promote living donation.

\section{Compliance with Ethics Guidelines}

Conflict of Interest Geir Mjøen and Hallvard Holdaas declare that they have no conflict of interest.

Human and Animal Rights and Informed Consent This article does not contain any studies with human or animal subjects, performed by any of the authors. 


\section{References}

Papers of particular interest, published recently, have been highlighted as:

- Of importance

•- Of major importance

1. Matas AJ, Smith JM, Skeans MA, Thompson B, Gustafson SK, Schnitzler MA, et al. OPTN/SRTR 2012 Annual Data Report: kidney. Am J Transplant. 2014;14 Suppl 1:11-44.

2. www.eurotransplant.org. Accessed August 12, 2014.

3. Merrill JP, Murray JE, Harrison JH, Guild WR. Successful homotransplantation of the human kidney between identical twins. J Am Med Assoc. 1956;160(4):277-82.

4. Fehrman-Ekholm I, Norden G, Lennerling A, Rizell M, Mjornstedt $\mathrm{L}$, Wramner $\mathrm{L}$, et al. Incidence of end-stage renal disease among live kidney donors. Transplantation. 2006;82(12):1646-8.

5. Fehrman-Ekholm I, Elinder CG, Stenbeck M, Tyden G, Groth CG. Kidney donors live longer. Transplantation. 1997;64(7):976-8.

6. Rosenblatt GS, Nakamura N, Barry JM. End-stage renal disease after kidney donation: a single-center experience. Transplant Proc. 2008;40(5):1315-8.

7. Mjoen G, Reisaeter A, Hallan S, Line PD, Hartmann A, Midtvedt $\mathrm{K}$, et al. Overall and cardiovascular mortality in Norwegian kidney donors compared to the background population. Nephrol Dial Transplant. 2012;27(1):443-7.

8. Lentine KL, Schnitzler MA, Xiao H, Saab G, Salvalaggio PR, Axelrod D, et al. Racial variation in medical outcomes among living kidney donors. N Engl J Med. 2010;363(8):724-32.

9. Wafa EW, Refaie AF, Abbas TM, Fouda MA, Sheashaa HA, Mostafa A, et al. End-stage renal disease among living-kidney donors: single-center experience. Exp Clin Transplant. 2011;9(1): $14-9$.

10. Okamoto M. Long-term renal function, complications and life expectancy in living kidney donors. World J Transplant. 2012;2(1):5-8.

11. Ibrahim HN, Foley R, Tan L, Rogers T, Bailey RF, Guo H, et al. Long-term consequences of kidney donation. N Engl J Med. 2009;360(5):459-69.

12. Cherikh WS, Young CJ, Kramer BF, Taranto SE, Randall HB, Fan $P Y$. Ethnic and gender related differences in the risk of end-stage renal disease after living kidney donation. Am J Transplant. 2011;11(8):1650-5.

13. Fournier C, Pallet N, Cherqaoui Z, Pucheu S, Kreis H, Mejean A, et al. Very long-term follow-up of living kidney donors. Transpl Int. 2012;25(4):385-90.

14. Lin J, Kramer H, Chandraker AK. Mortality among living kidney donors and comparison populations. N Engl J Med. 2010;363(8): 797-8.

15. Segev DL, Muzaale AD, Caffo BS, Mehta SH, Singer AL, Taranto SE, et al. Perioperative mortality and long-term survival following live kidney donation. JAMA. 2010;303(10):959-66.

16. Matuchansky C. Perioperative mortality and long-term survival in live kidney donors. JAMA. 2010;303(22):2248-50.

17. Bowman J. Perioperative mortality and long-term survival in live kidney donors. JAMA. 2010;303(22):2249-50.

18. Andreoni KA. "Live kidney donors live longer" and would you like to buy part of a bridge in Brooklyn? Arch Surg. 2010;145(7):701-2.

19. Garg AX, Meirambayeva A, Huang A, Kim J, Prasad GV, Knoll G, et al. Cardiovascular disease in kidney donors: matched cohort study. BMJ. 2012;344:e1203.

20. Mjoen G, Hallan S, Hartmann A, Foss A, Midtvedt K, Oyen O, et al. Long-term risks for kidney donors. Kidney Int. 2014;86(1):
162-7. Found increased risks of ESRD and death compared with a healthy control group during long-term follow-up.

21. Reese PP, Bloom RD, Feldman HI, Rosenbaum P, Wang W, Saynisch P, et al. Mortality and cardiovascular disease among older live kidney donors. Am J Transplant. 2014;14(8):1853-61. No increased risk of death compared with matched controls.

22. Reese PP, Bloom RD, Feldman PR, et al. (abstract) J Am Soc Nephrol. 2013; 23(suppl.):71A. Increased risk of ESRD compared with matched controls.

23.• Muzaale AD, Massie AB, Wang MC, Montgomery RA, McBride MA, Wainright JL, et al. Risk of end-stage renal disease following live kidney donation. JAMA. 2014;311(6):579-86. Increased risk of ESRD compared with healthy matched controls.

24. O'Dea DF, Murphy SW, Hefferton D, Parfrey PS. Higher risk for renal failure in first-degree relatives of white patients with end-stage renal disease: a population-based study. Am J Kidney Dis. 1998;32(5):794-801.

25. Lei HH, Perneger TV, Klag MJ, Whelton PK, Coresh J. Familial aggregation of renal disease in a population-based case-control study. J Am Soc Nephrol. 1998;9(7):1270-6.

26. Skrunes R, Svarstad E, Reisaeter AV, Vikse BE. Familial clustering of ESRD in the Norwegian Population. Clin J Am Soc Nephrol. 2014.

27. Gill JS, Tonelli M. Understanding rare adverse outcomes following living kidney donation. JAMA. 2014;311(6):577-9.

28. Kiberd B, Tennankore K. Kidney donation and risk of ESRD. JAMA. 2014;312(1):93.

29. Boudville N, Prasad GV, Knoll G, Muirhead N, Thiessen-Philbrook $\mathrm{H}$, Yang RC, et al. Meta-analysis: risk for hypertension in living kidney donors. Ann Intern Med. 2006;145(3):185-96.

30. Kasiske BL, Ma JZ, Louis TA, Swan SK. Long-term effects of reduced renal mass in humans. Kidney Int. 1995;48(3):814-9.

31. Williams SL, Oler J, Jorkasky DK. Long-term renal function in kidney donors: a comparison of donors and their siblings. Ann Intern Med. 1986;105(1):1-8.

32. Kasiske BL, Anderson-Haag T, Ibrahim HN, Pesavento TE, Weir MR, Nogueira JM, et al. A prospective controlled study of kidney donors: baseline and 6-month follow-up. Am J Kidney Dis. 2013;62(3):577-86.

33. Garg AX, Muirhead N, Knoll G, Yang RC, Prasad GV, ThiessenPhilbrook H, et al. Proteinuria and reduced kidney function in living kidney donors: a systematic review, meta-analysis, and meta-regression. Kidney Int. 2006;70(10):1801-10.

34. Kiberd BA. Estimating the long term impact of kidney donation on life expectancy and end-stage renal disease. Transplant Res. 2013;2(1):2.

35. Steiner RW, Ix JH, Rifkin DE, Gert B. Estimating risks of de novo kidney diseases after living kidney donation. Am J Transplant. 2014;14(3):538-44.

36. Steiner RW. More on the increased risks of young kidney donors. Am J Transplant. 2011;11(2):413.

37. Steiner RW. 'Normal for now' or 'at future risk': a double standard for selecting young and older living kidney donors. Am J Transplant. 2010;10(4):737-41.

38. Steiner RW, Spital A. Evaluating living kidney donor candidates with minor medical abnormalities. Kidney Int. 2007;72(3):378.

39. Steiner RW. Risk appreciation for living kidney donors: another new subspecialty? Am J Transplant. 2004;4(5):694-7.

40. Steiner RW, Gert B. A technique for presenting risk and outcome data to potential living renal transplant donors. Transplantation. 2001;71(8):1056-7.

41. Steiner RW, Gert B. Ethical selection of living kidney donors. Am J Kidney Dis. 2000;36(4):677-86.

42. Go AS, Chertow GM, Fan D, McCulloch CE, Hsu CY. Chronic kidney disease and the risks of death, cardiovascular events, and hospitalization. N Engl J Med. 2004;351(13):1296-305.

43. Mjoen G. The author replies. Kidney Int. 2014;85(6):1469. 
44. Delmonico F. A report of the Amsterdam forum on the care of the live kidney donor: data and medical guidelines. Transplantation. 2005;79(6 Suppl):S53-66.

45. Andrews PA, Burnapp L, Manas D, Bradley JA, Dudley C. Summary of the British Transplantation Society/Renal Association U.K. guidelines for living donor kidney transplantation. Transplantation. 2012;93(7):666-73.

46. Lamb KE, Lodhi S, Meier-Kriesche HU. Long-term renal allograft survival in the United States: a critical reappraisal. Am J Transplant. 2011;11(3):450-62. 